\title{
Checkpoint Blockade Immunotherapy
}

National Cancer Institute

\section{Source}

National Cancer Institute. Checkpoint Blockade Immunotherapy. NCI Thesaurus. Code C131060.

Any type of immunotherapy that targets and blocks the immune checkpoint pathways to activate immune responses against tumor cells. 